\title{
ACTIVITY RECORDED FROM THE STATOCYST NERVE OF PLEUROBRANCHAEA CALIFORNICA DURING ROTATION AND AT DIFFERENT TILTS*
}

\author{
J. WOOD and R. J. VON BAUMGARTEN \\ Mental Health Research Institute, University of Michigan, Ann Arbor, \\ Michigan 48104
}

(Received 14 February 1972)

\begin{abstract}
The statocysts of Pleurobranchaea californica lie lateral to the pedal ganglia. The thirteen receptor cells communicate with the cerebropleural ganglion via the thirteen axons of the statocyst nerve.

2. Each unit in the statocyst nerve of Pleurobranchaea responds over a limited range of positions.

3. Receptor adaptation is slow. Each position in the active range of a unit is coded by the same steady-state firing rate. Initial frequencies upon reaching a particular position differ, depending upon the direction of approach.
\end{abstract}

\section{INTRODUCTION}

THE STATOCYSTS of several gastropod molluscs have recently been extensively studied from several points of view. Light microscope studies by Dijkgraaf \& Hessels (1969) on Aplysia limacina and electron microscope studies by Wolff (1968) on several pulmonate gastropods; Quattrini (1967) on Helix aspersa; and Coggeshall (1969) on Aplysia californica indicate that there are relatively few receptor cells (eleven to fifteen in several pulmonates, thirteen in $A$. californica and A. limacina). These studies, as well as electrophysiological studies by Wolff (1970), indicate that each receptor cell communicates with the cerebral ganglion via a single axon.

A detailed study of the statocyst nerve of Pleurobranchaea californica was of interest for several reasons. Such a study is a necessary prelude to a study of the integration of statocyst nerve input to the cerebropleural ganglion somata which are large and easily identifiable in this species. The simplicity of the statocyst nerve in Aplysia with its relatively few axons, all first order, made it seem likely that an electrophysiological study on the closely related Pleurobranchaea would provide additional information not only on sensory coding but upon the physical phenomena involved in the stimulation of gastropod statocyst receptors as well. Finally, a study of Pleurobranchaea's statocyst was of interest from a comparative standpoint, because of the electrophysiological studies already conducted on pulmonate gastropods by Wolff (1970).

* This work was supported by Training Grant MH07417 from the N.I.M.H., United States Public Health Service and by N.I.H. Grant NB07753 to R. J. von Baumgarten. 
Pleurobranchaea's two statocysts lie lateral to the left and right pedal ganglia in the connective tissue sheath which encloses the central nervous system. Each statocyst nerve passes anteriorly for about $1 \mathrm{~cm}$ before entering the ipsilateral cerebropedal connective. The statocyst nerve of Pleurobranchaea can be much more easily dissected than that of Aplysia.

\section{MATERIALS AND METHODS}

Pleurobranchaea californica were maintained at $15^{\circ} \mathrm{C}$ for up to 2 weeks before use. For physiological study of the statocyst, a rotating platform was constructed by clamping a $\frac{5}{8}$-in. thick aluminum plate in a Bridgeport milling machine turntable (Fig. 1). The platform could be rotated through more than $360^{\circ}$ about a horizontal axis and the angular position read from a scale on the turntable as the platform was rotated by hand. A potentiometer connected to the platform at its axis of rotation generated a voltage position signal. In other experiments a slotted disc attached to the platform at its axis of rotation generated a position signal by interrupting a light beam falling on a photocell at $3^{\circ}$ intervals. A recording chamber cooled by circulating water was rigidly attached to the platform. For recording, the isolated central nervous system was pinned to a layer of Sylgard 184 on the transparent floor of the recording chamber. Illuminating the preparation by transmitted light facilitated dissection of the statocyst nerve. Throughout this procedure the preparation was bathed in sea water. A suction electrode similar to that described by Hartman \& Boettiger (1967) was used for recording from the statocyst nerve. It included a glass micropipette of $20 \mu$ tip dia., the approximate diameter of the statocyst nerve. The syringe used to apply suction was connected through a stopcock mounted on the platform. After drawing the severed end of the nerve into the electrode the stopcock was closed and disconnected from the syringe. The suction electrode assembly was held in a micromanipulator attached to a frame of $\frac{1}{2}$ in. aluminum rod mounted on the rotating platform. For added stability during tilting, a second brace of 1 in. aluminum rod on the same platform was fixed to the electrode holder with Pyseal cement.

Replacing the sea water with a 2 per cent solution of agar in sea water provided firm support for the preparation during rotation and prevented nerve stimulation by stretch duc to relative movement between the preparation and the suction electrode. At least $45 \mathrm{~min}$ elapsed between pouring the liquid agar $\left(37^{\circ} \mathrm{C}\right)$ over the preparation and recording. Standard recording methods were used. Movement artifacts during rotation were minimized by attaching the preamplifier to the platform and taping the recording leads to the platform to prevent their movement. Experiments were carried out at either $15^{\circ} \mathrm{C}$ or room temperature, without qualitative differences in the results. The analog position signal and action potentials were recorded on an Ampex FM tape recorder. Most records were analyzed on a PDP-8 computer. For each action potential, amplitude and time of occurrence with respect to a starting signal marking the end of rotation on a third channel of the tape recorder was measured and punched on paper tape, and a frequency histogram of action potential magnitudes was generated.

Subsequent data reduction employed an IBM 360 computer. The number of firings of each individual unit (recognized by amplitude from the amplitude histogram) was plotted against the time of firing, and scatter plots of interspike interval vs. time of occurrence were generated. Phase and latency relationships between different units active at the same position were also calculated.

Most experiments were also recorded on moving film. A few experiments were carried out on preparations in which most fibers of the statocyst nerve were intentionally crushed, leaving only a few functional units. This made interpretation of the film record simpler, since there were fewer units to separate visually. 


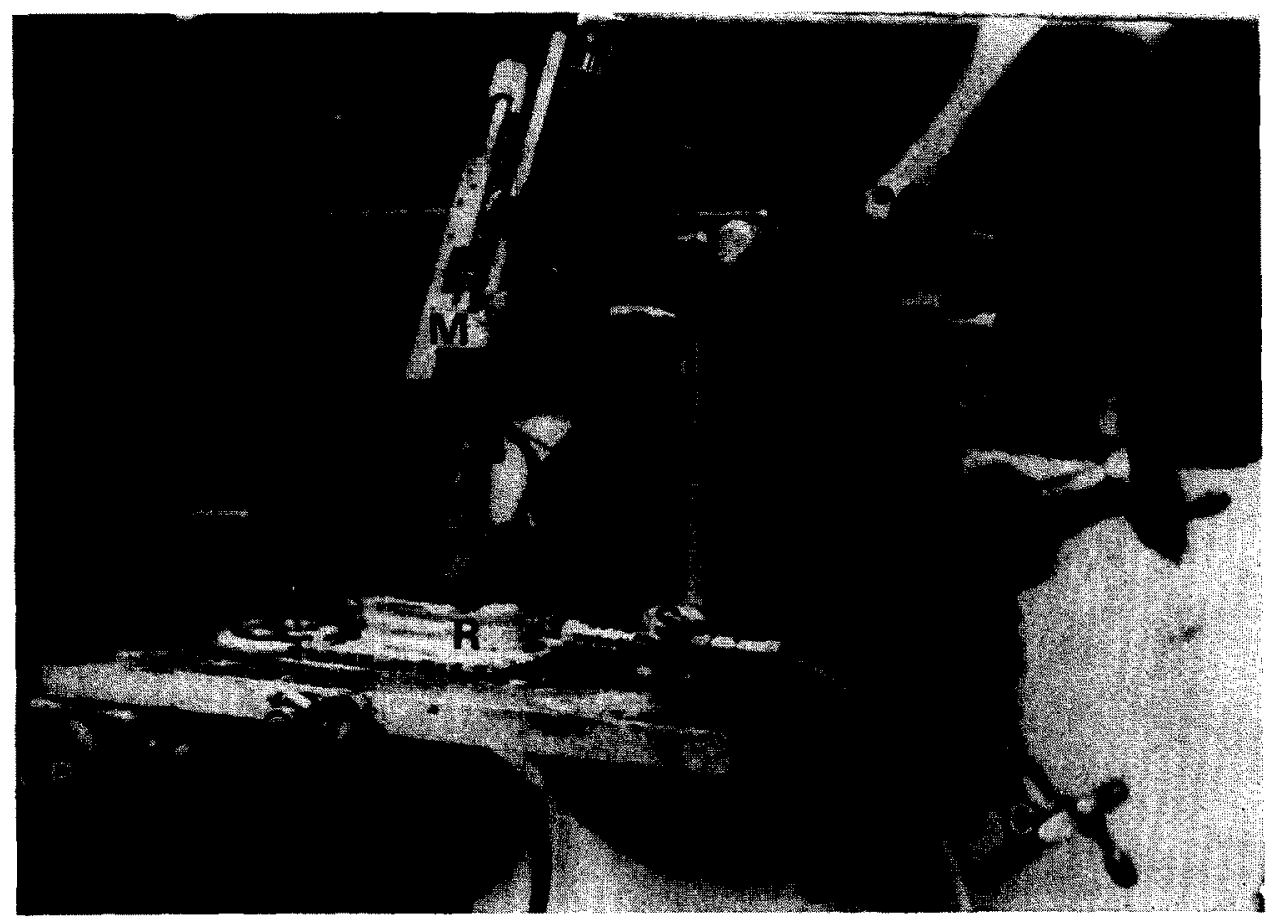

FIG. 1. The apparatus used for rotation and recording. E, suction electrode assembly; $\mathrm{B}$, electrode brace; $\mathrm{R}$, recording chamber; $\mathrm{T}$, rotating platform; $\mathrm{S}$, stopcock; $\mathrm{C}$, hand crank; $\mathrm{P}$, position indicator; $\mathrm{M}$, micromanipulator. 
It was established histologically that the statocyst of Pleurobranchaea was structurally similar to those of other gastropods. Tissue was fixed in buffered neutral formalin, and paraffin serial sections $(7 \mu)$ were stained with luxol fast blue and cresyl violet or with hematoxylin and eosin.

As in Aplysia (Dijkgraaf \& Hessels, 1969; Coggeshall, 1969), there are thirteen large receptor cells in each statocyst and thirteen axons in each statocyst nerve. Few statoconia remain in histological preparations. Under the dissecting microscope the statolith appears spherical with a diameter of about $150 \mu$ within the $200 \mu$ dia. statocyst, but slight pressure on the statocyst causes the statoconia to dissociate.

\section{RESULTS}

Recordings from the statocyst nerve showed several units spontaneously active at any one position. Continuous rotation demonstrated that each unit responds with maximum frequency soon after rotation into its active range. After continuous rotation past the position of maximum response, immediate rotation back to the position of maximum response elicits less activity than was initially elicited (Fig. 2). During rotation in small increments, each unit responded with

(a)

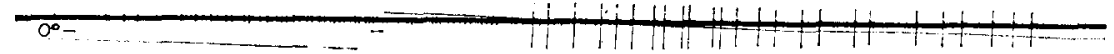

(b)

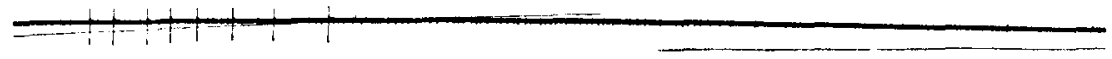

(c)

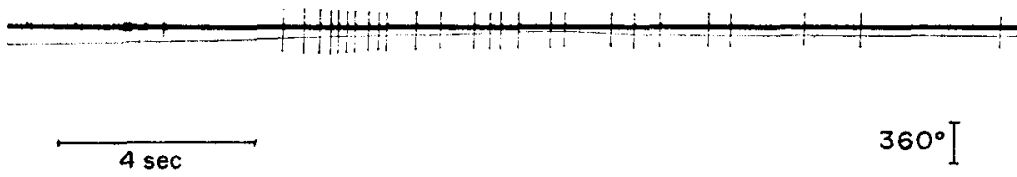

FIG. 2. Activity of a single fiber in the left statocyst nerve during rotation. Angular orientation is indicated by the initially lower trace with $0^{\circ}$ indicated by the horizontal mark on record (a). The steps in the trace occur at $180^{\circ}$ or upside down. (a) and (b) are continuous. (a) Clockwise rotation through the active range of the unit. (b) Counter-clockwise rotation through the active range of the unit. It became active $3 \mathrm{sec}$ after it stopped responding to clockwise rotation. (c) Response to rotation after a 2-min rest. Rotation into the range of the unit is initially counterclockwise, followed by clockwise rotation. Note that the response to counterclockwise rotation after rest is increased above that following the previous clockwise rotation and that rotation back to a position of previously higher activity does not cause an increase in activity.

increased frequency over approximately the same range of angular positions regardless of the direction of rotation about a given axis, but maximum numbers of impulses were recorded in particular positions. These positions differed when the preparation was rotated in opposite directions. Regardless of the direction of 
rotation, the maximum average frequency occurred close to the position at which the unit first became excited and average frequency declined beyond this point. Figure 3 illustrates the response to rotation about the animal's longitudinal axis recorded from a single unit in the left statocyst nerve. The preparation was
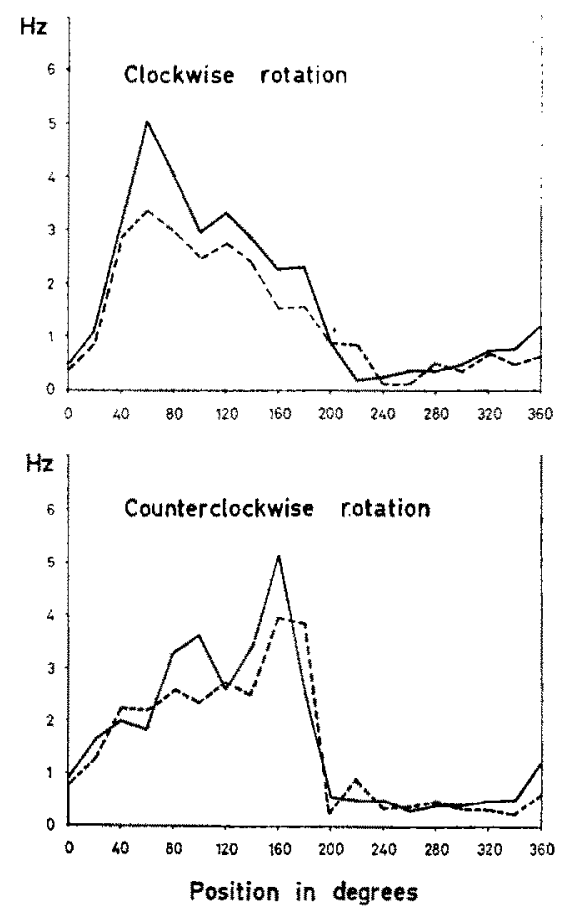

Frg. 3. Response of a single unit in the left statocyst nerve to rotation in $20^{\circ}$ steps about the longitudinal axis. The preparation remained in each position for $2 \mathrm{~min}$ and the initial frequency (impulses/sec) over the first $10 \mathrm{sec}$ is indicated by the solid line. Frequency during the period 110-120 sec is indicated by the dashed line.

rotated incrementally through $360^{\circ}$ clockwise (toward right side down) and then counter-clockwise through $360^{\circ}$. Maximum impulse frequencies over the 2-min period were recorded at orientations which differed by $80^{\circ}$.

Rotations through repeated sequences of positions yielded reproducible changes in the average frequency of single units. As in the case of rotations through $360^{\circ}$, the initial frequency at a particular position depended upon the direction from which the position was approached. The firing level finally approached a steady rate, which was characteristic of the position and the unit. In the experiment summarized in Fig. 4, the average impulse frequency over the 2-min period following rotation to the horizontal was lower when the direction of approach was clockwise than when the direction of approach was counter-clockwise. Counterclockwise rotation to the horizontal caused an immediate increase in average 


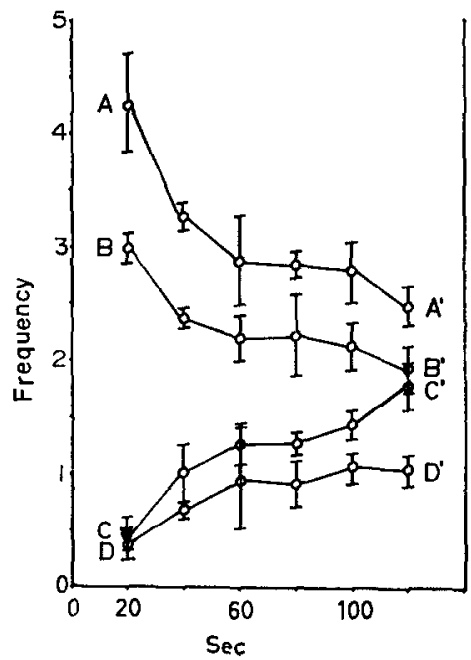

Fig. 4. Average frequency (impulses/sec) for successive 20 -sec intervals after $15^{\circ}$ rotations about the longitudinal axis. $A-A^{\prime}$, counter-clockwise rotation of $15^{\circ}$ from horizontal ; B-B', counter-clockwise rotation of $15^{\circ}$ to horizontal ; $C-C^{\prime}$, clockwise rotation of $15^{\circ}$ to horizontal; D- $\mathrm{D}^{\prime}$, clockwise rotation of $15^{\circ}$ from horizontal. Each point is the mean of three replications of the experiment. Vertical lines indicate \pm 1 S.D.

frequency, while clockwise rotation to the horizontal resulted in an immediate decrease in average frequency. During the 2 -min period following rotation to the horizontal, the higher frequency (following counter-clockwise rotation) decreased while the lower frequency (following clockwise rotation) increased (Fig. 4,B and C). Similar changes in frequency with time were seen after rotating the preparation from the horizontal. Clockwise rotation to $15^{\circ}$ right side down caused a decrease in frequency which then increased with time (Fig. 4D). Data from a different experiment using smaller increments of rotation is shown in Fig. 5. Tonic activity continues indefinitely, but adaptation is not a simple exponential decay of frequency over time.

No temporal relationships indicating synaptic interactions between receptor cells were found under the conditions of these experiments. Compound action potentials were occasionally seen. They did not occur regularly but occurred when two units, firing at different frequencies happened to fire simultaneously.

\section{DISCUSSION}

Rotation of the preparation in opposite directions in stepwise increments throughout the range of a given unit elicited maximum activity shortly after rotation into the range over which the unit responded. Wolff (1970) showed that for several pulmonate gastropods continuous rotation in opposite directions resulted in maximum activity at different positions, with the position of maximum 
(a)

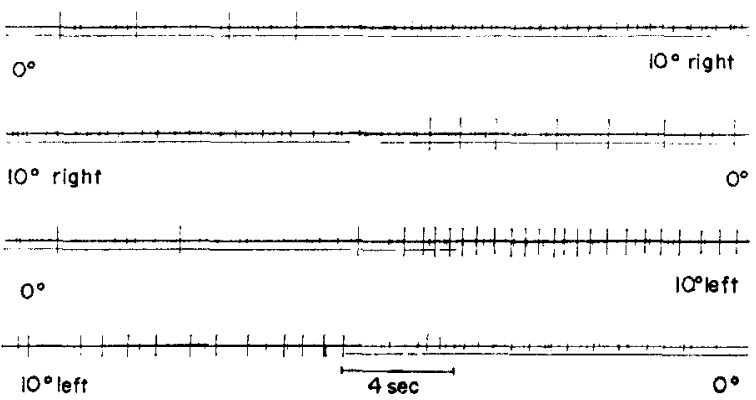

(b)
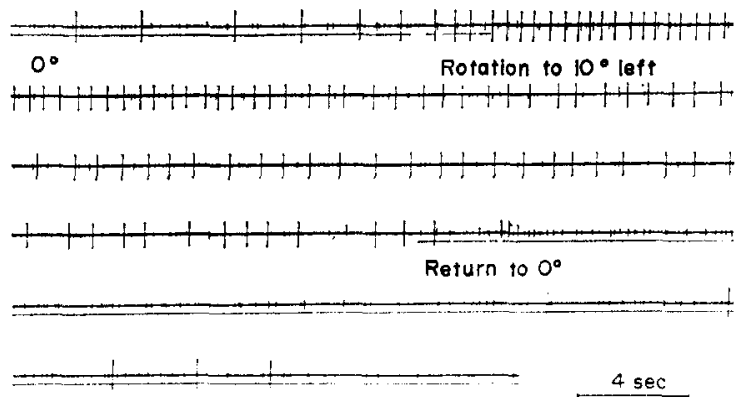

Fig. 5. Response of a single fiber in the left statocyst nerve to rotation in $10^{\circ}$ increments about the longitudinal axis. The spikes have been retouched. (a) Activity before, during and after rotation. The preparation remained in each position for $2 \mathrm{~min}$. Initial positions are indicated at the left, and final position at the right. The interruptions in the solid trace indicate rotation and occur at $3^{\circ}$ intervals. Note that the smaller units respond over a different range of positions than the large unit. (b) Adaptation after counter-clockwise rotation to $10^{\circ}$ left and clockwise to $0^{\circ}$. All records in (b) are continuous.

activity displaced in both cases so that it occurred closer to the point at which the unit first became active. Wolff also found that the range over which a particular unit responded shifted slightly depending upon the direction of rotation about a particular axis. Coggeshall (1969) observed that Aplysia statocyst receptor cells were indented by structures resembling other (possibly synaptic) structures present in its abdominal ganglion. It seemed possible that the directional sensitivity resulted from synaptic interaction between receptor cells. However, we did not observe phase or latency relations indicative of synaptic interactions between units with overlapping ranges. Intracellular recordings from the statocyst receptor cells will be necessary to finally resolve this question.

The time-course of frequency changes in a single unit when the same position is approached from opposite directions can probably be explained in terms of receptor adaptation, which also appears to be the cause of the apparent directional 
sensitivity. Regardless of the direction from which the active range of a unit is approached the unit begins firing with high frequency, but firing rate decreases by adaptation as the preparation continues to be rotated further into the active range. This interpretation assumes that the axons of the statocyst nerve in Pleurobranchaea are the first-order axons of receptor cells, as they are in Aplysia (Coggeshall, 1969; Dijkgraaf \& Hessels, 1969).

The responses of the Pleurobranchaea statocyst receptor cells resemble those of the lobster type II position receptors (Cohen, 1955) which show a positive dynamic response during rotation to a position of increased tonic activity and a negative dynamic response during rotation to a position of decreased tonic activity. A surprising result of our study was the long time-course of receptor adaptation. This may be necessary to provide an increased input after a position change during the relatively long period of compensatory movements. It also seems likely that the slow adaptation may be related to the presence of only one receptor type in Pleurobranchaea's statocyst, which must code information on position changes as well as absolute position, a situation distinctly different from that encountered in the otocysts of vertebrates, where several receptor types are present (Lowenstein \& Roberts, 1950). The few sense cells and the limited range of activity of each means that information from the statocyst is provided to the central nervous system via a set of labeled lines. The thirteen receptor cells in the statocyst seem to be labeled only by their geometrical position and not by their physiological properties.

Several possible transduction mechanisms have been suggested (Dijkgraaf \& Hessels, 1969; Wolff, 1970). Most rely upon the observations of Geuze (1968) on the structure of the cilia of the statocyst receptor cells of Lymnaea stagnalis. 'The basal feet of the cilia are directed radially toward the edges of the receptor cells. Wolff (1970) suggested, among other possibilities, that stimulation might involve the sliding of separate statoconia to the lowest part of the statocyst floor or that the statolith, made up of loosely bound statoconia might move, in an "almost amoeba like" viscous fashion to the lowest point in the statocyst. The cilia could then be deflected by the pressure of the statolith on the receptor cells beneath. The results obtained in these experiments are consistent with this interpretation although the dimensional considerations mentioned above make it seem more likely that stimulation of the receptor cells occurs before the statolith actually reaches the cilia. The rapid response of the statocyst after the onset of rotation suggests that currents caused by the displacement of statolymph by the slowly sinking statolith are involved in receptor stimulation.

Acknowledgements-We would like to thank Miss Barbara Friedman and Mr. Gordon Lyon for assistance in computer programming and Mr. Giulio Baldrighi for assistance in assembling the rotating platform and for drawing the illustrations.

\section{REFERENCES}

Catton W. T. (1970) Mechanoreceptor function. Physiol. Rev. 50, 297-317.

Coggeshall R. E. (1969) A fine structural analysis of the statocyst in Aplysia californica. F. Morph. 127, 113-132. 
CoHen M. J. (1955) The function of receptors in the statocyst of the lobster Homarus americanus. F. Physiol., Lond. 130, 9-34.

Dijkgraaf S. \& Hessels H. G. A. (1969) Úber Bau und Funktion der Statocyste bei der Schnecke Aplysia limacina. Z. vergl. Physiol. 62, 38-60.

GeuzE J. J. (1968) Observations on the function and the structure of the statocysts of Lymnaea stagnalis (L.). Neth. Y. Zool. 13, 155-204.

HaRtMan H. B. \& BoEtTiger E. G. (1967) The functional organization of the propusdactylus organ in Cancer irroratus Say. Comp. Biochem. Physiol. 22, 651-663.

Lowenstein O. \& Roberts T. D. M. (1950) The equilibrium function of the otolith organs of the thornback ray (Raja calvata). F. Physiol., Lond. 110, 392-415.

QUatTrini D. (1967) Osservazioni preliminari sulla ultrastruttura della statocisti dei molluschi gastropodi polmonati. Boll. Soc. ital. Biol. sper, 43, 785-786.

WolfF H. G. (1968) Elecktrische Antworten der Statonerven der Schnecken (Arion empiricorum und Helix pomatia) auf Drehreizung. Experentia (Basel) 24, 848-849.

WoLFF H. G. (1970) Statocystenfunktion bei einigen Landpulmonaten (Gastropoda) Verhaltens- und elektrophysiologische Untersuchungen. Z. vergl. Physiol. 69, 326-366.

Key Word Index-Statocyst; gravity receptors; rotation receptors; Pleurobranchaea californica. 